Physics

Physics Research Publications

\title{
Quantum phase transitions in disordered dimerized quantum spin models and the Harris criterion
}

D. X. Yao

E. W. Carlson
J. Gustafsson

A. W. Sandvik 


\title{
Quantum phase transitions in disordered dimerized quantum spin models and the Harris criterion
}

\author{
Dao-Xin Yao, ${ }^{1}$ Jonas Gustafsson, ${ }^{2,3}$ E. W. Carlson, ${ }^{4}$ and Anders W. Sandvik ${ }^{2}$ \\ ${ }^{1}$ State Key Laboratory of Optoelectronic Materials and Technologies, School of Physics and Engineering, Sun Yat-sen University, \\ Guangzhou 510275, China \\ ${ }^{2}$ Department of Physics, Boston University, 590 Commonwealth Avenue, Boston, Massachusetts 02215, USA \\ ${ }^{3}$ Theoretical Physics, Royal Institute of Technology, SE-10691 Stockholm, Sweden \\ ${ }^{4}$ Department of Physics, Purdue University, West Lafayette, Indiana 47907, USA
}

(Received 7 June 2010; revised manuscript received 7 August 2010; published 19 November 2010)

\begin{abstract}
We use quantum Monte Carlo simulations to study effects of disorder on the quantum phase transition occurring versus the ratio $g=J / J^{\prime}$ in square-lattice dimerized $S=1 / 2$ Heisenberg antiferromagnets with intradimer and interdimer couplings $J$ and $J^{\prime}$. The dimers are either randomly distributed (as in the classical dimer model), or come in parallel pairs with horizontal or vertical orientation. In both cases the transition violates the Harris criterion, according to which the correlation-length exponent should satisfy $\nu \geq 1$. We do not detect any deviations from the three-dimensional $\mathrm{O}(3)$ universality class obtaining in the absence of disorder (where $\nu \approx 0.71$ ). We discuss special circumstances which allow $\nu<1$ for the type of disorder considered here.

DOI: 10.1103/PhysRevB.82.172409

PACS number(s): 75.10.Jm, 75.10.Nr, 75.40.Mg, 75.40.Cx
\end{abstract}

Studies of effects of disorder (randomness) at phase transitions have a long history in statistical physics with the celebrated "Harris criterion" providing a guide for when disorder should be expected to be relevant, i.e., leading to changes in the critical exponents. ${ }^{1}$ The statement by Harris is that for a $d$-dimensional classical system, the exponent $\nu$ governing the divergence of the correlation length should satisfy $\nu$ $\geq 2 / d$ in the presence of disorder. If the exponent for an unperturbed (clean) system does not satisfy this relationship, then, in the presence of disorder, if the transition remains well defined (i.e., it is not smeared, with different transition points in different regions of the system ${ }^{2}$ ) a new universality class should obtain in which the relationship does hold. Alternatively, in some quantum systems the behavior instead becomes activated with exponential scaling instead of power-law singular behavior. ${ }^{3}$

The Harris criterion was originally derived based on a natural assumption of how the local critical temperature in some region of a classical system with random couplings can be directly related to local fluctuations in the average coupling strength (or impurity concentration). Consistency with a single critical temperature for the whole system (no smearing) then leads to the requirement $\nu \geq 2 / d .{ }^{1}$ This condition was later rederived using an alternative, more rigorous method, which allowed for extension to some quantum systems as well. ${ }^{4}$ The effective statistical-mechanics problem for a quantum system at temperature $T=0$ corresponds, through the Euclidean path integral, to a classical system in $d+1$ dimensions (under the assumption that quantum mechanical effects due to Barry phases can be neglected, which is not always the case ${ }^{5}$ ). Since disorder is introduced only in the original spatial dimension (corresponding to columnar disorder in the $d+1$-dimensional classical system), the dimensionality to use in the Harris criterion for a quantum system is presumed to be just $d$, not $d+1 .{ }^{4}$ Hence, for twodimensional quantum spin systems, which we will study in this paper, one would expect $\nu \geq 1$ at a quantum phase transition in the presence of disorder.
We will discuss quantum phase transitions in spin-1/2 dimerized Heisenberg antiferromagnets on the square lattice. A dimer consists of two nearest-neighbor spins coupled by a Heisenberg interaction of strength $J$. All spins belong to exactly one dimer, of which there are $N / 2$ for a lattice with $N=L \times L$ sites and $L$ even (and we use periodic boundary conditions). The dimers are coupled to each other through all the other nearest-neighbor bonds with weaker coupling $J^{\prime}$. The Hamiltonian is thus

$$
H=J \sum_{\langle i, j\rangle} \mathbf{S}_{i} \cdot \mathbf{S}_{j}+J^{\prime} \sum_{\langle i, j\rangle^{\prime}} \mathbf{S}_{i} \cdot \mathbf{S}_{j},
$$

where $\langle i, j\rangle$ is the set of dimers and $\langle i, j\rangle^{\prime}$ denotes the rest of the nearest-neighbor pairs. We will here consider disorder in the form of random configurations of the dimers, constructed in two different ways as illustrated in Fig. 1.

For a system with a regular (nonrandom) dimers, there is quantum phase transition as a function of the coupling ratio $g=J / J^{\prime}$. Examples include dimers arranged in columns ${ }^{6,7}$ or between the layers of a bilayer. ${ }^{8}$ According to standard sym-
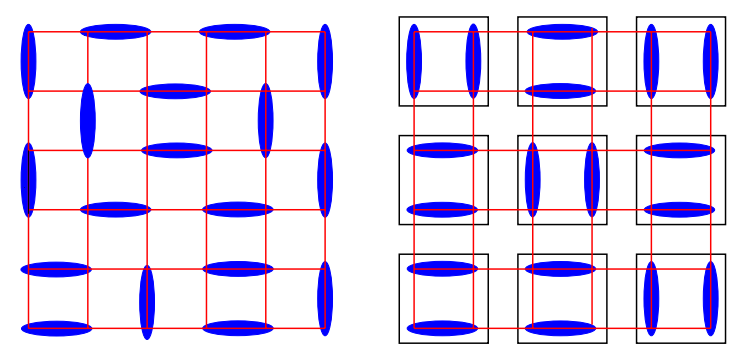

FIG. 1. (Color online) Dimerized systems with two types of configurational disorder. The dimers (shown as ovals) are spin pairs with interactions $J$ stronger than the interdimer couplings $J^{\prime}$. In the random dimer model (left) all close-packed dimer configurations are included whereas in the random plaquette system (right) a superlattice of $2 \times 2$ plaquettes has horizontal or vertical dimer pairs within the plaquettes. 
metry arguments the phase transition should be in the universality class of the $\mathrm{O}(3)$ (classical Heisenberg) model with $d=3$. There are, however, subtleties related to Berry phases and the way the continuum limit is taken in effective field theories, such as the $(2+1)$-dimensional nonlinear $\sigma$ model. ${ }^{9-12}$ Large-scale quantum Monte Carlo (QMC) calculations of bilayers and columnar dimers have given critical exponents in very good agreement with the expected universality class. Results for some other patterns, e.g., staggered dimers (where every second row of a columnar dimer pattern is shifted by one lattice spacing) are currently puzzling with either a different universality class obtaining ${ }^{7}$ or unexpectedly large corrections to scaling. ${ }^{13,14}$

Disorder can be introduced in these dimerized systems in many different ways. We are interested in systems with maintained SU(2) symmetry. Since $\nu<1(\approx 0.71),{ }^{15}$ disorder is expected to be relevant by the Harris criterion. On possibility is to dilute the system by removing a fraction of the spins at random. In general this will completely destroy the phase transition, however, because in the nonmagnetic phase the removal of a single spin leads to an uncompensated magnetic moment (the remaining spin of the dimer with a vacancy). For a finite concentration of vacancies the subsystem of liberated moments exhibits long-range order. One can circumvent this problem by removing whole dimers. ${ }^{16,17} \mathrm{~A}$ large-scale Monte Carlo study of an effective threedimensional classical model corresponding to this situation indicated a generic transition (at fixed dilution below the classical percolation threshold) satisfying the Harris criterion. $^{18}$

Here we introduce two different types of configurational disorders, illustrated in Fig. 1, as testing grounds for the Harris criterion and other challenging issues at quantum phase transitions in the presence of disorder. The random dimer model (RDM) is based on the ensemble of all dimer configurations, as in the classical dimer model. In the random plaquette model (RPM), we subdivide the lattice into $2 \times 2$ plaquettes and place two parallel dimers within all the plaquettes. Starting from a clean system of all horizontal dimers (a columnar configuration), we rotate a fraction $p$ of the dimer pairs by $90^{\circ}$. In this case $p$ is a well-defined measure of the degree of disorder in the system with maximum disorder at $p=1 / 2$ (which is the case we consider here, unless otherwise stated). In the RDM, on the other hand, there is no tunable impurity concentration or disorder strength. In addition, in this case the disorder is correlated, as the averaged dimer-dimer correlations decay as $1 / r^{2}$ in the closepacked dimer system. ${ }^{19}$ The prerequisites of the Harris criterion may then be violated in the RDM. ${ }^{20}$ I contrast, the RPM dimers are only locally correlated (within the individual plaquettes), which should be of no relevance in a coarsegraining sense. Our objective here is to investigate the role of correlated disorder in the RDM and to test the validity of the Harris criterion in both models.

We have performed quantum Monte Carlo simulations using the stochastic series expansion (SSE) method. ${ }^{21}$ Sufficiently low temperatures are used for obtaining ground-state results for lattices with $L$ up to 40 (using procedures for checking the $T \rightarrow 0$ convergence discussed in Ref. 22). We will discuss finite-size scaling of several quantities. The staggered structure factor is defined as

$$
S(\pi, \pi)=\frac{1}{N}\left\langle\left(\sum_{i=1}^{N} S_{i}^{z} \phi_{i}\right)^{2}\right\rangle=N\left\langle m_{s}^{2}\right\rangle,
$$

where $\phi_{i}= \pm 1$ is the staggered phase factor and $m_{s}$ is the sublattice magnetization. $S(\pi, \pi)$ should scale at a $d=2$ quantum-critical point as $L^{z-\eta}$, where the exponent $\eta$ $\approx 0.037$ in the $\mathrm{O}(3)$ universality class $^{15}$ and the dynamic exponent $z=1$. If the universality class changes due to the disorder, the new exponents are not known. The Binder ratio,

$$
Q_{2}=\frac{\left\langle m_{s}^{4}\right\rangle}{\left\langle m_{s}^{2}\right\rangle^{2}},
$$

is a dimensionless quantity with no size corrections (asymptotically) at criticality; $Q_{2}(L) \rightarrow$ constant at the critical point. We also study the spin stiffness, the second derivative of the ground-state energy $E(\phi)$ (per spin) in the presence of a boundary phase twist $\phi$

$$
\rho_{s}=\frac{\partial^{2} E(\phi)}{\partial \phi^{2}},
$$

which is obtained in the SSE simulations in the standard way using winding number fluctuations. ${ }^{23}$ It is scaling at criticality is only governed by the dynamic exponent $z ; \rho_{s} \sim L^{-z}$ in two dimensions.

We study disorder-averaged quantities. For each system size, at least several hundred configurations were used. We apply the standard finite-size scaling formalism, according to which a quantity $A$ should depend on the lattice length $L$ and the deviation from the critical point $g_{c}$ according to

$$
A(g, L)=L^{\kappa}\left(1+a L^{-\omega}\right) f\left[\left(g-g_{c}\right) L^{1 / \nu}\right],
$$

where $f$ is a nonsingular function, $\nu$ the correlation-length exponent, and $\kappa$ depends on the quantity considered, as discussed above. Here we have also included a subleading correction $\left(1+a L^{-\omega}\right)$, which in some cases is needed in order to obtain good fits to the data.

We have analyzed SSE data for the RPM and RDM in different ways, with and without scaling corrections, keeping all the exponents and the critical coupling ratio $g_{c}$ as variables in the fitting procedure or keeping some of them fixed to values obtained in other fits, using different sets of system sizes, etc. In all cases we find that $z=1$ can describe the data very well, and therefore conclude that the dynamic exponent is not changed by the disorder, in contrast to the random $\mathrm{O}(3)$ model (with rodlike impurities), ${ }^{18}$ for which $z \approx 1.3$ (which is well beyond the statistical uncertainties of a few percent for our result $z=1$ ). Surprisingly, all other exponents, as well, come out very close to their $d=3$ Heisenberg values. In a final stage of the analysis we therefore fix all the exponents to their best available $d=3 \mathrm{O}(3)$ values $^{15}$ and only adjust $g_{c}$ to optimize the fits. We consistently obtain good fits with values of $g_{c}$ that agree among the different quantities studied; our final estimates for the critical coupling ratios are $g_{c}=2.145 \pm 0.001(\mathrm{RDM})$ and $g_{c}=1.990 \pm 0.001 \quad(\mathrm{RPM})$. Figures 2 and 3 show some examples of data fits; for $\rho_{s}$ and $S(\pi, \pi)$ of the RDM without subleading corrections in Fig. 2 and for $Q_{2}$ and $S(\pi, \pi)$ of the RPM with subleading corrections in Fig. 3. 


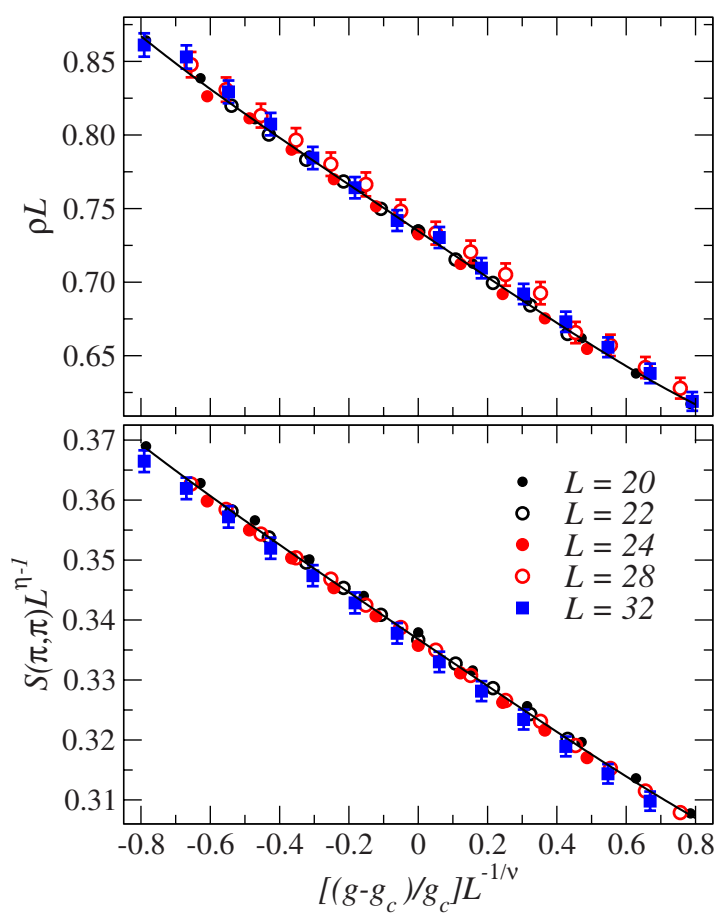

FIG. 2. (Color online) Finite-size scaling of the spin stiffness (top panel) and the staggered structure factor (bottom panel) of the RDM, using the $d=3$ Heisenberg exponents $(\eta=0.0375, \nu$ $=0.7115)$ and $g_{c}=2.145$. Where not shown, the error bars are smaller than the symbols. Note that the statistical errors for a given lattice size $L$ are correlated because the same random dimer configurations were used for all coupling rations $g$ and the sample-tosample fluctuations are larger than the QMC statistical errors.

The conclusion of this study is, thus, that the transitions in both the RPM and RDM violate the Harris criterion. It has been pointed out before that this criterion, in fact, contains several implicit assumptions that may make it inapplicable (or require extensions) for some systems. ${ }^{20,24}$ In addition, the criterion should really be written as $\nu_{\mathrm{FS}} \geq 2 / d,{ }^{4,24}$ where the finite-size correlation-length exponent $\nu_{\mathrm{FS}}$ is exactly the one extracted in scaling procedures such as those we have used above. The intrinsic correlation length can be detected using a modified procedure ${ }^{24}$ involving scaling relative to individual finite-size sample definitions of the critical point. The fact that our result shows unambiguously that $\nu_{\mathrm{FS}}<2 / d$ implies $^{24}$ that the sample-to-sample fluctuations of the critical point are smaller than assumed in the original derivations of the Harris criterion. We have studied these fluctuations and, indeed, find that they are very small (in fact, so small that it is difficult to study their size dependence quantitatively). The modified scaling procedure therefore also produces results consistent with the same $\mathrm{O}(3)$ exponents. It seems, therefore, that these exponents also are the intrinsic exponents are unchanged, remaining at their clean-system values.

A transition in violation of $\nu_{\mathrm{FS}} \geq 2 / d$ and unchanged exponents have also been found in the $d=2$ disordered bosonic Hubbard model, at the special multicritical point at the tip of the Mott lobes. ${ }^{25}$ There it was argued ${ }^{26}$ that the critical point does not depend on the disorder strength, which violates the

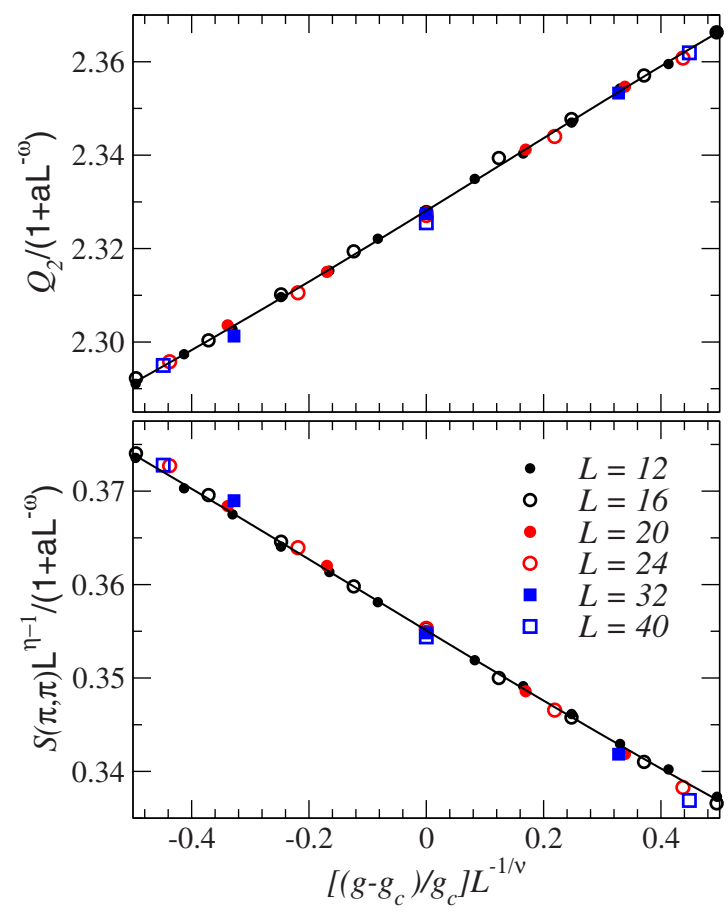

FIG. 3. (Color online) Finite-size scaling of the Binder ratio (top panel) and the staggered structure factor (bottom panel) or the RPM, using the $d=3$ Heisenberg exponents $(\eta=0.0375, \nu=0.7115)$ and the critical point $g_{c}=1.990$. The subleading exponent $\omega \approx 1$ in both cases and the prefactor $a \approx-0.5$ for $Q_{2}$ and $a \approx-0.1$ for $S(\pi, \pi)$. The error bars are at most on the order of the size of the symbols.

prerequisite of the Harris criterion of the possibility to drive the transition by tuning the disorder strength ${ }^{26}$ (although this is called into question by recent work ${ }^{27}$ ). This is the case also for our RDM, where there is no notion of disorder strength or concentration. In the RPM, there is, however, a clearly observable dependence on the probability $p$ characterizing the ratio of horizontal and vertical dimer pairs. At $p=0,1$, the critical value is the smallest, $g_{c}=1.909,6,7$ and the maximum value is $g_{c}=1.990$ at $p=1 / 2$, as reported above. The curve $g_{c}(p)$ is symmetric about the point $p=1 / 2$ that we analyzed above, and the local dependence on $p$ is particularly small there (but we do not know the exact form of $g_{c}$ versus $p$ ), which may explain the smallness of the sample-to-sample fluctuations in $g_{c}$ (which, according to Ref. 24 can account for $\left.\nu_{\mathrm{FS}}<2 / d\right)$. On the other hand, we have also studied $p$ $=1 / 4$ and also there find no changes in the exponents.

The RDM is a special case in the sense that the constrained disorder of close-packed dimers leads to dimerdimer correlations decaying as $1 / r^{2}{ }^{19}$ This represents the border-line case of disorder correlated according to a powerlaw $1 / r^{a}$, where for $a>2$ the usual Harris criterion should apply (in cases where the criterion is valid for uncorrelated disorder) and for $a<2$ a modified criterion was presented. ${ }^{28}$ This may be of no relevance here, however, since the usual Harris criterion is not valid for the uncorrelated RPM.

In summary, we have studied configurational disorder in dimerized square-lattice $S=1 / 2$ Heisenberg models. We find no change in universality class of the Néel to nonmagnetic 
quantum phase transition, in violation of the Harris criterion. While this criterion does not state the fixed point to which the disordered system flows, this point should, if the criterion is valid, satisfy $\nu_{\mathrm{FS}}>1$ for dimensionality $d=2$, which is ruled out by our results. Our study reinforces the notion that the Harris criterion can be violated. ${ }^{24}$ The transition does not represent the most likely scenario discussed in Ref. 24, where the exponents still would change due to the disorder. Such a case of unchanged exponents with $\nu_{\mathrm{FS}}<1$ has also been claimed in a previous study of hard-core bosons. ${ }^{25}$

We have not addressed the issue of Griffith's phases between the Néel and disordered (gapped) states, which, according to standard arguments, should generally be expected. They do not, however, have to influence the critical exponents, ${ }^{5}$ i.e., the unchanged exponents we have can very well apply also at a Néel-Griffith's critical point. In the case of the RDM, the clean staggered dimer configuration is part of its space and has a higher critical coupling than the average $g_{c}$ found here. ${ }^{7}$ Thus, one should expect arbitrarily large Néel domains in the nonmagnetic phase. The only point at which there may possibly not be a Griffith's phase is at $p$ $=1 / 2$ in the RPM, for which $g_{c}$ versus $p$ is a maximum.
Unless some particular regular dimer arrangement within the random plaquette space can be found for which $g_{c}$ is higher, the arguments for Griffith's singularities break down there. ${ }^{27}$ It should be possible to analyze Griffith's behavior in our models by calculating the temperature dependence of the uniform susceptibility and the field-induced magnetization. ${ }^{29}$

It is interesting to note that we do not observe any anomalous scaling behavior of the type recently observed in certain regularly dimerized systems such as the staggered dimer model. ${ }^{7,13}$ This could, in part, be explained by the fact that the numerical precision we have obtained here for disordered systems is not as high as in the studies of clean systems. One would at least naively expect potential effects of uncompensated Berry phases ${ }^{5}$ to be larger in systems with random dimer arrangements.

We would like to thank Tommaso Roscilde for useful discussions of Griffith's phases. D.X.Y. acknowledges support from Sun Yat-sen University, E.W.C. by Research Corporation and NSF under Grant No. DMR 08-04748, and A.W.S. by the NSF under Grant No. DMR-0803510.
${ }^{1}$ A. B. Harris, J. Phys. C 7, 1671 (1974).

${ }^{2}$ T. Vojta and R. Sknepnek, Phys. Status Solidi 241, 2118 (2004).

${ }^{3}$ D. S. Fisher, Phys. Rev. Lett. 69, 534 (1992); Phys. Rev. B 51, 6411 (1995).

${ }^{4}$ J. T. Chayes, L. Chayes, D. S. Fisher, and T. Spencer, Phys. Rev. Lett. 57, 2999 (1986).

${ }^{5}$ S. Sachdev, Quantum Phase Transitions (Cambridge University Press, Cambridge, 1999).

${ }^{6}$ M. Matsumoto, C. Yasuda, S. Todo, and H. Takayama, Phys. Rev. B 65, 014407 (2001).

${ }^{7}$ S. Wenzel, L. Bogacz, and W. Janke, Phys. Rev. Lett. 101, 127202 (2008).

${ }^{8}$ A. W. Sandvik and D. J. Scalapino, Phys. Rev. Lett. 72, 2777 (1994); L. Wang, K. S. D. Beach, and A. W. Sandvik, Phys. Rev. B 73, 014431 (2006).

${ }^{9}$ S. Chakravarty, B. I. Halperin, and D. R. Nelson, Phys. Rev. B 39, 2344 (1989).

${ }^{10}$ N. Read and S. Sachdev, Phys. Rev. Lett. 62, 1694 (1989).

${ }^{11}$ T. Einarsson and H. Johannesson, Phys. Rev. B 43, 5867 (1991).

${ }^{12}$ A. V. Chubukov, S. Sachdev, and J. Ye, Phys. Rev. B 49, 11919 (1994).

${ }^{13}$ F.-J. Jiang and U. Gerber, J. Stat. Phys. (2009) P09016.
${ }^{14} \mathrm{~S}$. Wessel (private communication).

${ }^{15}$ M. Campostrini, M. Hasenbusch, A. Pelissetto, P. Rossi, and E. Vicari, Phys. Rev. B 65, 144520 (2002).

${ }^{16}$ A. W. Sandvik, Phys. Rev. Lett. 89, 177201 (2002); 96, 207201 (2006).

${ }^{17}$ O. P. Vajk and M. Greven, Phys. Rev. Lett. 89, 177202 (2002).

${ }^{18}$ T. Vojta and R. Sknepnek, Phys. Rev. B 74, 094415 (2006).

${ }^{19}$ M. E. Fisher, Phys. Rev. 124, 1664 (1961).

${ }^{20}$ H. R. da Cruz and R. B. Stinchcombe, J. Phys. C 19, 3555 (1986).

${ }^{21}$ A. W. Sandvik, Phys. Rev. B 59, R14157 (1999).

${ }^{22}$ A. W. Sandvik, Phys. Rev. B 66, 024418 (2002).

${ }^{23}$ A. W. Sandvik, Phys. Rev. B 56, 11678 (1997).

${ }^{24}$ F. Pázmándi, R. T. Scalettar, and G. T. Zimányi, Phys. Rev. Lett. 79, 5130 (1997).

${ }^{25}$ J. Kisker and H. Rieger, Phys. Rev. B 55, R11981 (1997).

${ }^{26}$ F. Pázmándi and G. T. Zimányi, Phys. Rev. B 57, 5044 (1998).

${ }^{27}$ L. Pollet, N. V. Prokof'ev, B. V. Svistunov, and M. Troyer, Phys. Rev. Lett. 103, 140402 (2009).

${ }^{28}$ A. Weinrib and B. I. Halperin, Phys. Rev. B 27, 413 (1983); A. Weinrib, ibid. 29, 387 (1984).

${ }^{29}$ T. Roscilde and S. Haas, Phys. Rev. Lett. 99, 047205 (2007). 